\title{
Autonomía, legitimidad y legalidad: en torno de un antiguo conflicto entre las facultades de filosofía y de derecho ${ }^{1}$
}

\author{
Autonomy, Legitimacy and Legality: \\ About an Ancient Conflict Between \\ Philosophy and Law Schools
}

Maurício Keinert ${ }^{*}$

Recepción y evaluación de propuesta: 20/09/2015.

Aceptación: 28/12/2015.

Recepción y aceptación final: 10/03/2016.

Resumen: Se trata de un comentario al artículo "Autonomía moral y legitimidad democrática" de Jan- R. Sieckmann en el que se pretende discutir principalmente el vínculo que éste instituye entre los conceptos de autonomía y autonomía individual. El comentario se dirige a demostrar que tal vínculo no es obvio ni tampoco natural mediante el análisis de dos autores de la filosofía moderna que han estudiado el asunto, Immanuel Kant y Jean-Jacques Rousseau. A partir de esto, intenta también discutir cuestiones vinculadas a la relación entre legalidad y legitimidad, sea en la moral, sea en el derecho y en la política, que estarían por detrás del concepto de autonomía tal como es pensado por Kant. Por último, se plantean algunos problemas para pensar la legitimidad del derecho y de la democracia.

Palabras clave: Autonomía, legitimidad, legalidad.

Doctor en Filosofía, Universidad de San Pablo, Brasil.

Correo electrónico: mkeinert@usp.br

1 Traducción de María Valentina Risso 
Maurício Keinert

\begin{abstract}
This work is a commentary on the article "moral autonomy and democratic legitimacy" from Jan-R Sieckmann. Here I pretend to discuss mainly the link that the author establishes between the concepts of autonomy and individual autonomy. This work pretends to demonstrate that this link is neither obvious nor natural, and it will be shown through the analysis of two authors of the modern philosophy such as Immanuel Kant and Jean-Jacques Rousseau. Starting from this point, I also pretend to discuss different issues related to the connection between legality and legitimacy, either in the field of morals, or law and politics, which would be behind the concept of autonomy as it is thought by Kant. Finally, I present some problems in order to think the legitimacy of law and democracy.
\end{abstract}

Keywords: Autonomy, legitimacy, legality.

El artículo de Jan- R. Sieckmann, "Autonomía moral y legitimidad democrática", tiene dos pretensiones que desearía destacar desde el principio. En primer lugar, pretende reflexionar sobre la legitimidad del sistema democrático por medio del concepto de autonomía. Investiga las fuentes de este concepto en la modernidad, por medio de filósofos como Rousseau y principalmente Kant, para luego desarrollar una estimulante discusión contemporánea sobre la posibilidad de trabajar este concepto como criterio de legitimidad del poder político. Sieckmann enumera de manera notable en sus notas una sucesión de importantes teóricos que estudiaron minuciosamente la cuestión, como Rawls, Habermas, Alexy y Dworkin, entre otros. En segundo lugar, el artículo también está dirigido a plantear una discusión más filosófica, en el sentido de cuestionar la validez misma del concepto de autonomía y, para ello, promoverá un análisis anclado en importantes nombres de la Kant-Forschung, sobre lo que denomina de una paradoja que se encontraría en la formulación de este concepto. Gran parte del artículo será destinado a esta discusión, sin embargo finalmente, veremos que no se trata de una paradoja en sentido fuerte, pues el autor afirma haber llegado a una solución del problema. Desearía realizar mi comentario poniendo en consideración la cuestión de la paradoja de la autonomía para, luego, desarrollar algunas observaciones sobre el uso del concepto para pensar la legitimidad del poder político, más específicamente, de la democracia. 
Autonomía, legitimidad y legalidad: en torno de un antiguo conflicto...

\section{Ambigüedad estructural}

Existe una ambigüedad estructural en el texto de Sieckmann que es problemática y se refiere a la manera como, a partir de su argumentación, él analiza el concepto de autonomía. Desde el principio, sin decirnos exactamente la razón, el autor identifica tal concepto con el de autonomía individual. Además de resultar problemática esta reciprocidad entre los conceptos y de no estar debidamente justificada, principalmente desde el punto de vista de la filosofía kantiana, ella es reduccionista: se trata de una visión extremadamente liberal ${ }^{2}$ del concepto de individuo, visto de manera inflexible como portador de intereses determinados de forma puramente mecánica. Es en estos términos que la paradoja de la autonomía nos es presentada. Por otra parte, al exponer su solución para la paradoja, Sieckmann va gradualmente incluyendo en el concepto elementos que no estaban presentes anteriormente como, por ejemplo, la propia idea de ponderación de argumentos o de pretensiones normativas que están presentes en su concepción de autonomía (que no estaría relacionada con la paradoja). Por lo tanto, si comenzamos la lectura del artículo teniendo en cuenta una idea de autonomía cuya característica principal solo se refiere a los intereses propios de un individuo, al final de este mismo artículo el concepto de autonomía tiene en cuenta el concepto de intersubjetividad, de posibles criterios universalistas y la consideración de buenos argumentos. Aunque el autor continua tratando el concepto de autonomía individual hasta el final, esta ambigüedad en cómo construye su argumento parece debilitar su misma posición.

Podría argumentarse contra esta clave de lectura que Sieckmann partiría de una estructura moderna de autonomía, presente en Kant

2 Considero perfectamente posible defender una posición liberal con relación al concepto de individuo, no es este el problema. Sieckmann parece trabajar en el inicio de su artículo, sin embargo, con una concepción de individuo próxima a aquellas teorías de la "rational choice", lo que supondría una concepción de la estructura de la democracia que reproduciría la del mercado. No obstante, ésta no parece ser la posición de Sieckmann al final de su artículo. 
y Rousseau. Veamos. Ya en las primeras líneas de su introducción expone la siguiente tesis: como cualquier otra forma de poder político, el derecho debe ser legítimo, es decir, debe justificar el ejercicio de su poder y hasta incluso la utilización de la fuerza. Para el autor, el criterio para razonar sobre tal forma de legitimación es la autonomía de los individuos que están sujetos a ese poder y seguidamente afirma que ese es el único criterio para pensar la legitimación del poder político. Ahora bien, ¿por qué esta primacía de la autonomía individual ${ }^{3}$ ? Sieckmann afirma entonces:

De otro modo, sostener que existe una justificación del poder político sobre la base de un criterio independiente de la autonomía individual implicaría que algunos individuos puedan estar sujetos al poder político a pesar de que puedan con razón objetarlo. Pero si alguien pudiera con razón rechazar el poder político, no existiría una justificación o legitimación de este poder.

Esta lectura supone que de hecho es el individuo y, por lo tanto, su voluntad y sus intereses, el principal criterio de legitimación del poder político y no exactamente el concepto de autonomía. De este modo, tendríamos la institucionalización del poder político con el objeto de hacer posible la mayor libertad para el desarrollo de los individuos y de sus intereses. Considero que este es el verdadero presupuesto que guiará la lectura que el autor realiza de Rousseau y de Kant.

A continuación presento la primera cuestión que me interesaría desarrollar: el hecho de que la filosofía moderna política haya partido de la cuestión del individuo no la convierte necesariamente en liberal, mucho menos cuando estamos tratando de autores como Kant y Rousseau. Sin embargo, no parece ser esta la posición de Sieckmann. Del primer filósofo, el autor conserva principalmente el famoso problema planteado en el inicio del Contrato Social, el de "encontrar una

3 Cabe preguntarse incluso por qué la primacía de la autonomía, ya que la historia, la historia de la filosofía, la ciencia política, etc. nos muestran una variada gama de posibilidades de justificación o de legitimación del poder político que no se refiere al concepto de autonomía. 
Autonomía, legitimidad y legalidad: en torno de un antiguo conflicto...

forma de asociación que proteja y defienda con toda la fuerza común a la persona y a los bienes de cada asociado y en virtud de la cual cada uno obedezca a sí mismo y permanezca tan libre como antes"4. Del segundo, recoge el hecho de que el poder legislativo representa la voluntad unida de un pueblo y que, siendo así, todo el derecho debe provenir de este poder y debe ser imposible que una ley de este implique un acto injusto contra alguien, pues, según Kant, solamente la voluntad unificada de todos, en que cada uno y todos deciden lo mismo para todos y cada uno, puede legislar. Estos dos aspectos, presentes en Rousseau y Kant, son importantes para nuestro autor, que concluye:

Por consiguiente, el problema de legitimar el poder legislativo es el de cómo la existencia de las leyes puede resultar compatible con la libertad de cada uno y cómo, a su vez, es posible dar cuenta de leyes tales que puedan contar con el consentimiento de cada uno y, así, volverse legítimas.

Esta es la idea de consentimiento de cada uno de los individuos en relación al poder que será el "núcleo de la legitimidad democrática". Sin embargo, una vez más es evidente que el criterio de apreciación de Sieckmann se refiere a la voluntad misma del individuo más que precisamente al concepto de autonomía.

En la medida en que la introducción del artículo llega a este núcleo, se plantean algunos problemas. El principal se refiere al hecho de que, tanto en el derecho como en la política, la ley, o su aplicación, tiene un carácter vinculante y, por lo tanto, puede entrar en oposición a la libertad de aquellos que están sujetos a ella. En ese sentido, la instauración del imperio de la ley llevaría a esos individuos a perder su libertad, o al menos, a verla transformada. Esa cuestión, que será desdoblada en otras, podría ser, entonces, abordada por nosotros de la siguiente manera: ya hay una concepción de libertad aquí tratada, un presupuesto duro, no siempre explícito, que convertirá el concepto de autonomía en problemático: la idea de que cada uno de los individuos dará su consentimiento de acuerdo con sus propios intereses. Este

${ }^{4}$ Rousseau, J-J., Du Contrat Social, Paris: Pléiade, 1964, pág. 360. 
presupuesto no me parece armonizar con los conceptos de autonomía tal como lo encontramos en Rousseau y, principalmente, en Kant.

\section{Autonomía moral: Kant y Rousseau}

Una de las virtudes del artículo de Sieckmann es la de encontrar en el interior de la filosofía moderna elementos constitutivos del debate político y jurídico contemporáneo. En ese sentido, la reconstrucción del concepto de autonomía en Rousseau y en Kant propuesta por el autor es importante, pese a que padezca de un problema de análisis. El problema, como dijimos, se refiere a la reciprocidad entre el concepto de autonomía moral y el de autonomía individual, presupuesta por el artículo, pero que, sin embargo, no es abordada de forma directa. Pues bien, ¿será que el concepto de autonomía forjado a lo largo del siglo XVIII se limita al concepto de autonomía individual, concebido específicamente en el siglo $\mathrm{XX}^{5}$ ? Entonces, ¿será que el concepto de autonomía individual haría posible que hablásemos de autonomía en Kant y Rousseau sin señalar las especificidades de cada uno de ellos? Sieckmann, sin embargo, aproxima los dos filósofos por medio del concepto de individuo que, a su vez, lo llevará al concepto de autonomía individual.

Cuando Rousseau plantea el problema de la relación entre el individuo y la asociación de individuos, que se convertirá en un cuerpo político, él tiene en mente el pasaje de la libertad natural a la libertad convencional de estos últimos. Tal pasaje le permitirá afirmar que cada uno, uniéndose a todos, no obedece sino a sí mismo, pues, al formar parte ahora del cuerpo político soberano, el individuo, en tanto ciudadano, al obedecer la voluntad general, obedece a sí mismo. Esto significa, y es lo importante, que cada uno cede su libertad natural, y sus derechos naturales, en favor de la unidad del cuerpo político, volviendo a todos, desde el punto de vista moral y político, ciudadanos autónomos y, por lo

5 Cf. O'Neill, O. "Autonomy, Plurality and Public Reason", en: Brender, N. Krasnoff, L. (eds.), New Essays on the History of Autonomy, Cambridge, Cambridge University Press, 2004, págs. 182-3. Hay una traducción de este artículo en portugués: O'Neill, O., "Autonomia, pluralidade e razão pública", Cuadernos de filosofia alemana, V. 19, n. ${ }^{\circ}$ 1, 2014. 
Autonomía, legitimidad y legalidad: en torno de un antiguo conflicto...

tanto, iguales y libres desde el punto de vista de la convención alcanzada por el pacto social. De esta manera, le es posible afirmar al ginebrino que "en lugar de la persona particular de cada contratante, este acto de asociación produce un cuerpo moral y colectivo, compuesto por tantos miembros como votos tiene la asamblea, el cual recibe por ese mismo acto su unidad, su yo común, su vida y su voluntad"6.

Cómo hablar entonces de autonomía individual en el Contrato Social, pues, en rigor, solo sería posible hablar de autonomía por medio del acto de asociación, cuando la persona particular se convierte en un miembro de la voluntad general y cuando, por lo tanto, ella pasa a formar parte de un cuerpo colectivo, moral y político. La idea de soberanía, por lo tanto, será central para Rousseau, ya que es con la autonomía de los individuos, asociados en un cuerpo político único, que él podrá ocuparse de una relación que tenga en cuenta dos puntos de vista distintos: la pasividad y la actividad. Si el cuerpo político es activo como soberano y pasivo como Estado, el pueblo es, en cuanto una comunidad de ciudadanos, activo y, mientras está sometido a las leyes del Estado, sujeto y pasivo. Esta doble relación nos permite pensar entonces el concepto de autonomía: en primer lugar, él se refiere a la unidad de la asociación que es soberana y promulga las leyes del Estado, la forma institucional de esta soberanía; en segundo lugar, los ciudadanos como partícipes de esta soberanía son agentes de estas leyes, pero, por ser sujetos y pasivos, están también sometidos a ellas. De este modo, son literalmente legisladores y destinatarios de estas leyes.

Es evidente el esfuerzo de Rousseau en subrayar el acto de asociación. En la medida en que el individuo se asocia, se convierte en un ciudadano de la República y es en el interior de esta comunidad, esto es, como individuo partícipe de esta comunidad política, que él legisla para sí mismo y para todos los demás ciudadanos. En este sentido, al acentuar la actividad de la asociación política, Rousseau resalta el elemento de la igualdad política. Todos somos autónomos y, por lo tanto, iguales. Esto permite que todos legislen para todos en el interior de la República. Por otra parte, desde la perspectiva de la soberanía, todos

${ }^{6}$ Rousseau, J-J., op. cit., pág. 361. 
deben volverse libres, por lo tanto, todos deben ser obligados a adherir al contrato por medio de la voluntad general, teniendo su voluntad particular, como individuo, constreñida por la voluntad general, lo que hace que el cuerpo político se mantenga unificado y cohesionado. De esta forma, el concepto de autonomía en Rousseau está estrechamente vinculado al concepto de soberanía del cuerpo político y cuando se habla de individuo, se habla de aquel individuo que se asoció por medio del pacto social y que, al hacerlo, rechaza su derecho y su libertad naturales, que se refieren a su fuerza particular, como individuo particular. Por lo tanto, cuando se habla de autonomía es imposible pensar en un individuo que en su actividad legislativa no esté asociado al cuerpo político, es decir, un individuo que dejó de lado sus intereses particulares en beneficio de la voluntad general. La idea de autonomía en este caso no es, de ninguna manera, recíproca a la idea de autonomía individual.

Es interesante la forma que Kant encuentra para reconstruir el concepto de autonomía de Rousseau, dando al acto de asociación un significado a priori y trascendental. Sin embargo, la cuestión se vuelve más compleja en la medida en que esta actividad requiere ser analizada por medio de un doble punto de vista, atinente a los usos de la razón práctica: el moral, por un lado, y el jurídico-político, por el otro ${ }^{7}$. En la Fundamentación de la metafisica de las costumbres, Kant pretenderá investigar, desde el principio, la acción humana por medio de dos maneras distintas: una en que la acción será analizada por la intención y, por lo tanto, teniendo valor en sí misma; otra en que será analizada por el objeto de la acción, aquello que ella pretende lograr por sí misma como un medio. En el primer caso, habría, para Kant, una acción

7 Tengo en cuenta la posición de Kant en sus Lecciones de derecho natural del año 1784 conocidas como Naturrecht Feyerabend. En este curso, así como algunos años más tarde en la Metafísica de las Costumbres, la idea de moral tendría en cuenta tanto elementos de la ética como del derecho. En este sentido, trabajo con el siguiente enfoque: la Fundamentación de la metafisica de las costumbres no es solo una obra sobre la ética, sino antes bien sobre la moral (aun cuando no desarrolle explícitamente los elementos del derecho, desarrollados en la Naturrecht Feyerabend). 
Autonomía, legitimidad y legalidad: en torno de un antiguo conflicto...

por deber, en el segundo caso, una acción conforme al deber. En relación al primer tipo, el autor afirma: "una acción por deber tiene su valor moral no en el propósito que por medio de ella se quiere alcanzar, sino en la máxima por la cual ha sido decidida, no depende, pues, de la realidad efectiva del objeto de la acción, sino meramente del principio del querer, según el cual ha sucedido la acción, prescindiendo de todos

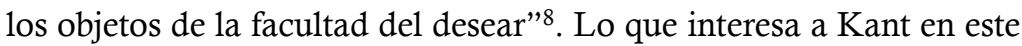
caso es el hecho de que el valor moral de la acción no se encuentra, pues, en el propósito a ser alcanzado por medio de ella y, de este modo, no depende de la realidad efectiva del objeto de la acción, sino que se encuentra en la máxima según la cual ha sido resuelta y en el principio del querer.

Pues bien, en este momento de la Fundamentación, la preocupación del filósofo está puesta en el criterio para decidir el valor moral de una acción que, según él, reside en su principio formal, a priori, y no en su principio material, a posteriori, vinculado al objeto de la acción, o a un determinado fin a ser conseguido. Tal criterio necesitará ser decidido entonces por medio de la regla que guía la acción, su máxima. Tal vez sea posible decir que este camino seguido por la argumentación kantiana se refiera al valor incondicionado de la buena voluntad que, en este momento, se confunde con el valor moral. Siendo ello así, cualquier principio material vinculado a un determinado fin $\mathrm{u}$ objeto será siempre condicionado y el análisis se orientará necesariamente al principio formal- el principio del querer. El paso siguiente será el de identificar este principio formal a una actividad de la voluntad, vinculada, a su vez, al respeto a la ley. Cuando afirma que "el deber es la necesidad de una acción por respeto a la ley", Kant vincula aquella actividad de la voluntad a la posibilidad de descubrir una razón o un fundamento (Grund) de la acción que no esté vinculado a su vez a cualquier objeto o principio empírico.

De ello concluye la importante afirmación de la Fundamentación: "una acción por deber tiene que excluir por completo todo influjo de

8 Kant, I., Fundamentação da metafísica dos Costumes, San Pablo, Barcarolla, 2009, AK.VII, págs. 399-400. 
la inclinación y con ella todo objeto de la voluntad, no queda, pues, otra cosa que pueda determinar la voluntad sino objetivamente la ley $\mathrm{y}$, subjetivamente, el puro respeto a esa ley práctica, y por lo tanto, la máxima de obedecer siempre a esa ley, aún con perjuicio de todas mis inclinaciones" ". Pues bien, el valor moral de la acción, como valor incondicionado y que, por lo tanto, no reside en ningún elemento empírico, solo se puede vincular a la determinación de la ley. Por lo expuesto, es posible decir que esta determinación debe ser entendida a través de dos puntos mencionados en la exposición hecha hasta aquí: en primer lugar, se trata de afirmar que el criterio para decidir el valor moral de una acción requiere el principio formal del querer que, a su vez, se identifica con la actividad de la voluntad; en segundo lugar, se pretende afirmar también que esta actividad de la voluntad se encuentra en la búsqueda por el fundamento (Grund) a priori de su acción. De la manera como entendemos la estructura del argumento de Kant, es posible sostener lo siguiente: cuando él establece la relación entre máxima y ley práctica afirmando, en un primer momento, que aquella es el principio subjetivo y ésta el principio objetivo del querer, la actividad de la voluntad de hallar un fundamento para la acción se confunde con un procedimiento de comparación de la forma de la máxima con la universalidad de la ley. Tal procedimiento, racional, permite la constatación, entonces, en un segundo momento, del valor moral de la acción en su determinación.

Esta actividad de comparación de la máxima con la ley, realizada por el agente racional, es un punto que es fuente de controversia tanto por parte de los comentadores como de los pensadores contemporáneos. Específicamente en una cuestión que nos parece decisiva para la discusión que aquí se traba. Se relaciona exactamente con lo que está en discusión en el proceso de comparación de la máxima con la ley y de cuál perspectiva se adopta allí: ¿una perspectiva individual o una perspectiva en que el individuo se encuentra inserto en el interior de un todo moral? Es conocido el artículo de Rüdiger Bittner, "Máxi-

9 Ibidem. pág. 129 (AK. VII; págs. 400-401). 
Autonomía, legitimidad y legalidad: en torno de un antiguo conflicto...

mas"10, en el que su interpretación de este concepto permite dos lecturas aparentemente antagónicas, aunque de gran interés. El primer punto de análisis de Bittner se refiere a la diferencia que él realiza entre los conceptos de máxima y de propósito. Para diferenciarlos, propone que el segundo estaría vinculado a ciertos elementos particulares del querer, reglas que no tienen ninguna importancia desde el punto de vista del valor moral, como la de un individuo que elige para sí mismo salir a cenar todos los lunes a la noche. Otra cosa muy diferente sería el concepto de máxima, que tendría en cuenta lo que el comentador llama de experiencia de vida, es decir, aquello que se refiere a la totalidad de la vida de un individuo, como el hecho que una determinada persona tenga en la avaricia el elemento organizativo de sus acciones, es decir, de su modo de vivir. Esta interpretación tiene, en mi opinión, el valor de esclarecer un aspecto no siempre observado en el interior del kantismo, es decir, que las máximas están, en cuanto organizan un conjunto de reglas subjetivas de acción, insertas en un determinado contexto de vida ético ${ }^{11}$.

Sin embargo, el segundo punto tratado por Bittner parece desautorizar esta interpretación en el siguiente aspecto: la totalidad de la vida implícita en la máxima, ya universalizada, por lo tanto, se refiere apenas a la totalidad de las acciones de un determinado individuo, sin necesariamente tomar en consideración el otro. En este sentido, la totalidad que la máxima comprende se refiere únicamente a las acciones de un único individuo, más específicamente de su voluntad:

Esto significa que la voluntad, por intermedio de sí misma, quiere que ambos (la regla universal, y el hacer particular) sean uno, y que por lo tanto, ese hacer sea querido al mismo tiempo como universal, y precisamente debido a su universalidad. En la autolegislación, la propia disposición de la ley y la sumisión

${ }^{10}$ Bittner, R., "Máximas", Studia Kantiana, n. ${ }^{\circ}$ 05, 2004, págs. 7-25.

${ }^{11}$ Sobre esto, Bittner es enfático: "Que los seres humanos estén siempre en relaciones morales (sittlichen) concretas, y en su contexto entiendan y determinen sus vidas, como sabia Aristóteles, no es olvidado tampoco en Kant, antes bien está en la base de la reflexión moral: sus máximas son ésta autodeterminación del individuo a partir de la experiencia de su mundo". Ibídem, pág. 14. 
a la misma no se dividen en dos grupos de propósitos; si así fuese, ella no sería más autolegislación (Selbstgesetzgebung) ${ }^{12}$.

Por lo tanto, el modo como el comentador entiende el concepto de autonomía en Kant es sui generis, pues apunta a un concepto de autonomía de la voluntad que no está limitada críticamente por la razón, pero es potencializada por la razón. La autonomía es de este modo la reconciliación de la voluntad consigo misma, como voluntad particular y universal de un mismo individuo, o incluso en las palabras de Bittner:

Según su contenido, la máxima es ya de la más alta universalidad, a saber, principio de toda una vida. Ella es un particular únicamente por intermedio de su forma- de valer sólo en razón de mi decisión para $m i$ vida, por lo tanto por intermedio de su subjetividad ${ }^{13}$.

Aunque Bittner pretenda dar complejidad al concepto de subjetividad en Kant, exponiendo que la máxima posee un contenido vinculado a la experiencia de vida de un determinado individuo, su solución para la relación entre máxima y ley, así como el modo como entiende el concepto de autonomía, no parecen encontrar refugio en la letra y en el espíritu kantiano ${ }^{14}$.

Si retomáramos la reconstrucción del argumento de la Fundamentación que hacíamos, podemos afirmar que el concepto de respeto a la ley es algo que nos puede ayudar en la tarea de esclarecer esta difícil relación entre la máxima y la ley. En primer lugar es posible afirmar que el respeto proporciona, mientas es un sentimiento autoproducido por medio de un concepto de la razón, el reconocimiento de la determinación de la voluntad por la ley, es decir, la conciencia de la subordinación de la voluntad a la razón. Por lo tanto, la consciencia de la subordinación de la voluntad a la ley es el efecto de la determinación de la ley

12 Ídem, pág. 23.

${ }^{13}$ Ídem, págs. 23-4.

${ }^{14}$ Principalmente por el hecho de que no encontramos en el artículo de Bittner lo que sería el criterio del valor moral de una acción. ¿Qué es lo que, por ejemplo, diferenciaría una acción por deber de una acción contraria al deber, si la máxima ya es un principio universal? 
Autonomía, legitimidad y legalidad: en torno de un antiguo conflicto...

que, desde el punto de vista subjetivo, es entendida como el efecto del proceso de universalización de la máxima. La idea de un sentimiento autoproducido por la razón plantea, a mi entender, un proceso que es doble: la comparación de la máxima con la universalidad de la ley y la consiguiente determinación de la voluntad por la ley. Es este "doble movimiento" el que explicaría la relación entre la subjetividad de la máxima y la objetividad de la ley, aquello que se refiere a la propia actividad de la voluntad de hallar un fundamento a priori de su acción y de verse determinada de forma racional, es decir, es a partir de él que podemos afirmar la posibilidad de que una voluntad sea una razón práctica. En segundo lugar, es necesario prestar atención al hecho de que el sentimiento autoproducido del respeto es la representación de lo que Kant llama menoscabo o derogación del amor a mí mismo y, por lo tanto, presupone un movimiento de renuncia parcial del yo.

Ahora bien, ¿cuál sería el sentido de señalar este menoscabo del amor a mí mismo y este movimiento de descentramiento del yo? En mi opinión, estas dos características demuestran que en la medida en que el sujeto racional representa la ley moral, tal representación presupone inmediatamente dos características: el proceso de comparación de la máxima con la ley y, aún más, con la forma universal de la ley $\mathrm{y}$, por consiguiente, la posibilidad de encontrar un fundamento de determinación de la acción y, al mismo tiempo, el hecho de que todos los otros seres racionales poseen la misma capacidad y, por lo tanto, son sujetos interesados en la representación que el individuo hace de la ley. Si pensáramos la representación de la ley por medio del concepto de deliberación, podemos afirmar entonces que al comparar la máxima con la ley, la razón inmediatamente se pregunta por la universalidad de la forma de la máxima, y tal universalidad, cuando es positiva, supone el hecho de que todos los otros seres racionales (exigencia de totalidad de la razón) prestarían su consentimiento a tal acción. En este sentido, al representar la ley y actuar por medio de esta paralegalidad ${ }^{15}$, el individuo delibera en el interior de un todo moral que, a su vez, se confundirá con el valor moral incondicional de su acción, pues

${ }^{15} \mathrm{~N}$. del T.: el autor utiliza el término "legiformidade" que en inglés sería el equivalente "law-like". 
es él el que dará legitimidad a la regla de la acción. Es en este movimiento de representación (o de deliberación) que podemos reconocer el concepto kantiano de autonomía, pues al representar la ley, al hacer la comparación de la máxima con la ley, este individuo legisla por medio de su actividad racional para sí mismo y para todos los otros seres racionales, colocándose de este modo en la posición de legislador y de destinatario de la ley.

Al comportarse así, este individuo no está actuando por medio de un cálculo de intereses propios, por el contrario, al representar la ley, la mayoría de las veces como afirma Kant, estará actuando contra sus propias inclinaciones en caso de que decida proceder de acuerdo con la ley. Concluimos una vez más que, tanto en Kant como en Rousseau, el concepto de autonomía no se ciñe al concepto de autonomía individual. Sin embargo, y este es el punto importante, creo que hay aquí una diferencia importante con relación a Rousseau que debe ser investigada: todos los individuos, por ser autónomos y, por lo tanto, interesados, son también un fin en sí mismo ${ }^{16}$ y por esta cualidad, son seres portadores de dignidad, son seres racionales. Si por lo tanto Rousseau tiene una posición republicana fuerte, en la medida en que la voluntad particular del individuo debe ser coaccionada por la voluntad general, en el proceso de la decisión de la asamblea ${ }^{17}$, en Kant esta relación tendrá que ser reconsiderada por la representación de la ley también en su vertiente político-jurídica.

\section{Autonomía política: Kant y el uso público de la razón}

En su opúsculo "Respuesta a la pregunta: ¿qué es la ilustración?", Kant establece una importante relación para pensar la representación de la ley jurídica: se ocupa de la distinción que hace allí entre el uso privado y el uso público de la razón. Si Rousseau, en el Contrato Social,

16 Tengo en cuenta aquí la formulación del imperativo categórico conocida como "fórmula de la humanidad", en la que todos los hombres son considerados como fines en sí mismos.

${ }^{17}$ Cf. Rousseau, J.-J., op. cit., principalmente el capítulo VII del Libro I. págs. 362-4. 
Autonomía, legitimidad y legalidad: en torno de un antiguo conflicto...

pretende diferenciar la figura del hombre de la del ciudadano, en que el primero, aun pensando de forma diferente, debe ser obligado a colocarse en la posición del segundo, es decir, a actuar y a pensar de acuerdo con la voluntad genera $1^{18}$, Kant, en su artículo, parece reorganizar esta relación entre el pensar (o racionalizar) y la obediencia. La idea de que el sujeto en la medida en que hace uso privado de su razón debe obedecer a una cierta autoridad en el interior de una determinada comunidad (el oficial que obedece las normas del ejército; el sacerdote o pastor que obedece las normas de la Iglesia; el ciudadano y contribuyente que obedece las normas del Estado) y, desde un punto de vista universal, debe hacer uso público de su razón, cuestionando las mismas normas, establece una relación entre ciudadanos, en que la obediencia tiene como condición de posibilidad el libre pensar. Es porque hago uso público de mi razón que obedezco las leyes constitucionales, por ejemplo. Pues bien, hacer uso de la razón pública tiene en cuenta algunas características importantes del concepto de autonomía.

En primer lugar, es necesario reflexionar sobre la propia forma de la constitución republicana, es decir, de la ley jurídica. Esta, así como la ley moral, requiere la misma relación autónoma entre el legislador y el destinatario. La ley tiene como condición de legitimidad la posibilidad de interpretarla y en este sentido, la posibilidad de encontrar razones o fundamentos (Grunds) para legitimarla o deslegitimarla ante el público. Estas razones necesitan, por lo tanto, ser presentadas públicamente y, en varios textos, Kant afirmará que el soberano tiene el deber de comportarse de acuerdo con lo que dice la voluntad general. En este sentido, la voluntad general, en términos kantianos, presupone la idea de una deliberación pública, y este es el elemento legitimador de la obediencia. Yo obedezco porque puedo criticar. Desde el punto de vista de la letra y del espíritu de la ley, obedezco a la Constitución porque esta se encuentra en disputa, es decir, puede ser interpretada de varias formas, afirmándola o no. En el artículo "Respuesta a la pregunta", Kant utiliza un ejemplo importante, el de un determinado grupo eclesiástico que establece para su comunidad una sujeción eter-

${ }^{18}$ Ídem. pág. 363. 
na e incesante sobre cada uno de sus miembros y pregunta si tal ejemplo se ajustaría con el concepto de Ilustración (Aufklärung). Él mismo responde que no, pues la Ilustración presupone algún tipo de progreso en que una generación lega a las siguientes mejores condiciones para su propia Ilustración. Y presenta en seguida un criterio de evaluación de la ley: "La piedra de toque de todo cuanto puede acordarse como ley para un pueblo se cifra en esta cuestión: ¿acaso podría un pueblo imponerse a sí mismo semejante ley?".

Se puede decir que la cuestión planteada aquí se refiere de nuevo a un procedimiento de comparación entre la ley positiva y la universalidad de la razón: ¿la ley positiva hace posible el florecimiento de un debate público de todos los interesados, la deliberación, o ella lo obstruye? Y debe tenerse en cuenta que incluso si esa ley ha sido establecida por la autoridad suprema del Parlamento o por un tratado de paz, ella resultará injusta desde el punto de vista de la Ilustración. Aquí entramos en un segundo ámbito importante para nuestra discusión, que se refiere a la naturaleza coercitiva de la ley positiva. No hay duda que Kant asume la posición que la ley jurídica, por su carácter legal, siempre será coercitiva. Sin embargo, tal coerción debe legitimarse de forma pública, de tal manera que ella por lo menos no impida el debate público. En este sentido, aunque la noción de justicia esté vinculada a la legalidad de la ley, este fragmento del opúsculo parece mostrar claramente que ella está relacionada también a la legitimidad, resultado del uso público y autónomo de la razón.

Dicho esto, la perspectiva de Kant parece ser bastante distinta de aquella adoptada por Rousseau. En este último, el individuo es obligado a volverse libre, es decir, a pasar de su condición de homme portador de opiniones propias a la condición de ciudadano forzado por la posición (verdadera) de la voluntad general. Tal presión es necesaria para la cohesión de la voluntad general, que no puede admitir ninguna forma de interés que sea diferente del suyo. En Kant, por el contrario, al distinguir (ya desde el punto de vista moral) la forma de la materia de la ley, la cohesión de la voluntad general sucede dada la posibilidad misma del uso público de la razón, es decir, de la deliberación autónoma del público. Como en la relación subjetiva de la máxima con la 
Autonomía, legitimidad y legalidad: en torno de un antiguo conflicto...

ley moral, en la que hay un proceso de comparación entre las formas de una con la otra y en que se pregunta si tal regla es válida para todos los seres racionales, desde el punto de vista político-jurídico, la deliberación se refiere al hecho de si la forma de una determinada ley positiva permite o no la deliberación de todos los posibles interesados al respecto. Este proceso es el que garantizará la legitimidad de la ley y, sobretodo, su respeto que aquí se traduce por la idea de coerción. Por lo tanto la coerción, al no estar envuelta en la relación binaria entre opinión y verdad, permite la amplificación de las diferentes voces que hacen uso público de su razón ${ }^{19}$.

\section{IV. ¿Paradoja de la autonomía?}

La rápida reconstrucción del concepto de autonomía tanto en Rousseau como en Kant nos ayuda a comprender mejor un punto importante: en los dos filósofos, el concepto de autonomía está vinculado a la idea de una actividad. Si en Rousseau tal actividad es representada por el acto del contrato, en el que hay una transición de la libertad natural en dirección a la libertad convencional del cuerpo político, en Kant ese pasaje sucede en la actividad de la representación de la ley moral, del propio juicio práctico, como también en la actividad de deliberación pública, por medio del uso público de la razón. En ambos casos, el modo como la ley es entendida permite una doble interpretación: al mismo tiempo en que ella es coerción (sea ética, interior, sea legal, exterior), ella es también expresión de la libertad. En este sentido, es posible afirmar que la autolegislación de la ley es la condición de obediencia de la misma y en ambos casos tenemos un elemento paradigmático: en el

${ }^{19}$ Esta idea parece ser fundamental tanto en "Respuesta a la pregunta: ¿qué es la Ilustración? Como en "Idea de una historia de una historia universal en sentido cosmopolita". En ambos artículos esta paradoja de la se ley se presenta: cuanto más una sociedad es coaccionada por leyes jurídicas, aún más ella puede modificar su modo de pensar público y, por lo tanto, ser libre desde el punto de vista de la Ilustración. Una lectura contemporánea de esta paradoja puede ser vista en el capítulo III de Faktizität und Geltung de Jürgen Habermas. 
caso de Rousseau, la ley significará un acto de soberanía, la reafirmación constante de la instauración originaria de la república de todos los ciudadanos políticos. En Kant, como tenemos dos puntos de vista, hay en primer lugar una transición a un todo moral y, en segundo lugar, el pase y constante reafirmación de la constitución republicana, es decir, la reafirmación de la justicia para todos los ciudadanos.

Me interesaría entonces discutir dos afirmaciones de Sieckmann sobre la paradoja de la autonomía, poniendo en consideración las dos grandes cuestiones discutidas en las partes precedentes. En la primera, el autor afirma:

La idea de autonomía como autolegislación parece tener una estructura paradojal. Entendida como autolegislación, la autonomía parece exigir que la validez de las normas dependa de su reconocimiento, quiere decir, de que sus destinatarios, en cuanto agentes autónomos, reconozcan que estas normas son válidas. Pero, por otro lado las normas pretenden vincular u obligar a sus destinatarios, y ¿cómo puede una persona estar vinculada por normas cuya validez depende de que ella misma, como destinataria, las reconozca como válidas? $\mathrm{Si}$, por el contrario, las normas son válidas con independencia del juicio individual, ¿Cómo se puede sostener que los individuos sean autónomos? ¿Si ellos no determinan que normas son válidas, no están autolegislando?

Si tenemos en cuenta las posiciones de Kant reconstruidas aquí, esta afirmación, en mi opinión, no tiene sentido por dos razones. La primera se refiere a la propia estructura del modo en que Kant entiende el concepto de ley, o incluso, de la representación de la ley. Se trata de una actividad racional en que es posible reconocer dos momentos (analíticos) precisos: el primero se refiere a la comparación de la máxima con la ley, es decir, la comparación de la máxima con la forma universal de la razón. Tal comparación se orienta por el fundamento de determinación de la ley. Un segundo momento en el que, en la medida en que tal fundamento es encontrado (la propia condición de universalidad de la ley), tenemos la determinación misma de la regla de la acción. Dos puntos así deben ser explicitados: 1. Esta actividad 
Autonomía, legitimidad y legalidad: en torno de un antiguo conflicto...

no presupone una potencialización de la voluntad por la razón (como Bittner sostiene), sino, por el contrario, una subordinación y una delimitación de la voluntad a la razón, en la medida en que ella se vuelve, en este caso, enteramente racional. 2. Esta actividad de representación de la ley es una actividad presupuesta en todo ser racional, por lo tanto, todo ser racional es o puede ser autónomo y, por esta condición, todos están alcanzados en relación con cualquier regla de acción. Estos dos puntos nos llevan a pensar en una relación que no es meramente empírica entre el interés mecánico del individuo y la ley, sino, por el contrario, se trata de una relación en que el interés individual no es puesto necesariamente en cuestión pero, sí el interés práctico -el que está verdaderamente en cuestión en la Fundamentación-.

La segunda afirmación de Sieckmann está más ligada a este último punto abordado:

El dilema de la autonomía se suscita porque la autolegislación implica que la validez de una norma depende de la voluntad, es decir, de un acto de reconocimiento por parte de la persona a quien ésta se dirige. La validez deber ser entendida en sentido normativo, es decir, la validez de una norma implica que esta se debe aplicar y cumplir. Desde luego esta elección deber ser razonable, es decir, debe cumplir con los requisitos de una decisión razonable; pero no puede derivase de normas preexistentes que hayan sido establecidas sobre la base de una moral o de la razón práctica. Ahora, si una norma es válida solamente a causa de la decisión de sus destinatarios, cabría entonces decir que no puede ser vinculante para ellos, y por lo tanto tampoco ser válida en un sentido normativo. La paradoja de la autonomía surge, entonces, del hecho de que la decisión autónoma, por una parte deber ser autodeterminada y no puede derivarse de normas preexistentes, aunque, por otra parte, pretende establecer una norma válida, lo que presupone que hay razones normativas por las cuales la norma en cuestión debe ser considerada válida y, finalmente, vinculante para sus destinatarios. Por lo tanto, las decisiones autónomas no pueden ser por completo libres: tienen que estar limitadas normativamente. El primer requisito hace depender la validez de las normas de la aceptación individual, aun cuando el 
segundo implique que tal propiedad es, en algún sentido, independiente de la aceptación de sus destinatarios.

Dos cuestiones pueden ser planteadas: en primer lugar, no hay duda que la validez de una norma es el resultado de la decisión de una voluntad, sin embargo aquí nos ocupamos de una voluntad que está siendo limitada críticamente por la razón. Lo que pretendí defender hasta este momento es que tal limitación se refiere a un proceso en que tenemos dos momentos analíticamente distintos: el proceso de universalización de la máxima, la comparación de su forma con la forma de la ley, es decir, la pregunta si ella es o no universal (en términos kantianos, la pregunta por su fundamento de determinación), y el proceso de determinación de la acción. Ambos procesos nos llevan a pensar en un doble punto de vista del concepto de ley kantiana: su legitimidad y su legalidad. Esta doble perspectiva se confunde con los elementos de coerción y libertad de la ley. En la medida en que este proceso requiere una universalidad, supone que están implicados en esta posición todos los seres racionales. De este modo, se pretende afirmar, como dijimos anteriormente, que lo que hace que sea posible validar la autonomía es exactamente el proceso de legitimación y la legalidad de la norma.

Ahora bien, desde el punto de vista de la ley político-jurídica, el concepto de decisión tiene que ser pensado teniendo en cuenta el concepto de deliberación ${ }^{20}$. Al hacer uso público de la razón, todos los posibles afectados en una determinada comunidad pueden cuestionar la legitimidad de una determinada ley positiva. Tal cuestionamiento nada dice respecto a la validez de la constitución en sí misma y su forma (republicana), pero sí respecto a ciertas características de una ley positivada, si ella permite el uso público de los implicados o no. En este sentido, desde el punto de vista kantiano, lo que dará la legitimidad al contrato originario será la propia idea de justicia que nace

${ }^{20}$ Para esta discusión, sigo en gran parte el artículo de Manin, B. "On Legitimacy and Political Deliberation". Political Theory, Vol. 15, n. ${ }^{\circ}$ 3, 1987. págs. 338-368. Para nuestros propósitos, Manin pretende defender un déficit de deliberación en la teoría política del Contrato Social de Rousseau y, con esto, un déficit de legitimación. Sin embargo, al identificar a Kant con una figura ultraliberal, ignora el valioso concepto del uso público de la razón. 
Autonomía, legitimidad y legalidad: en torno de un antiguo conflicto...

de la ponderación de argumentos en el interior del debate público $y$ de la legalidad de la ley. Como sostuvimos previamente, si con Rousseau el acto originario del contrato es la reafirmación del concepto de soberanía de la República, en Kant, la actividad de la deliberación y de legislación será la reiteración de la idea de justicia. Sin embargo aquí aparece un elemento importante: al mismo tiempo en que hay rastros republicanos en el concepto jurídico de ley kantiana, tenemos también elementos liberales, en la medida en que la posición de todos los afectados debe ser tenida en cuenta. De esta forma, pensar la autonomía en sentido político en Kant es pensar, primeramente, en un proceso de deliberación en el que hay un elemento de soberanía y, en segundo lugar, un elemento en que diferentes posiciones individuales deben ser avaladas por la ley. En Kant, esos dos elementos parecen confirmar dos puntos precisos: la deliberación de todos los implicados y la necesidad de preservar la posición de la minoría.

\section{Consideraciones finales}

Para concluir este comentario, tal vez sea importante explicitar algunos puntos que fueron abordados anteriormente. En primer lugar, creo que si Sieckmann pudiese trabajar con esa doble cara de la ley que intentamos mostrar en la actividad misma de representación de la ley en Kant, tanto desde el punto de vista moral en general como desde el punto de vista jurídico y político en particular, él podría retomar las principales características de su propuesta para de este modo pensar la solución de la paradoja de la autonomía, es decir, la intersubjetividad, la ponderación de argumentos y criterios universales. Y esto para solucionar algo que, en mi opinión, no quedó enteramente claro en su artículo. Se trata, en realidad, de clarificar dos puntos: el primero se refiere a ¿qué hace legítima la decisión de una determinada disputa política o jurídica? El autor utiliza el ejemplo de la prohibición o no de fumar en lugares públicos. Ahora bien, lo que me parece que daría legitimidad al proceso de decisión sería la posibilidad de todos los implicados que hicieren uso público de su razón, es decir, deliberasen. Si este principio no fuera tenido en cuenta cuando pensamos el 
concepto de autonomía, tendremos siempre, o casi siempre, la victoria del más fuerte en la decisión final. Y el derecho tendrá, en tanto poder político, su legitimación reducida a la legalidad y, por consiguiente, al concepto de fuerza o de poder. Esta cuestión de la diferenciación entre el poder y la razón o la propia autonomía de los ciudadanos, es el segundo punto que, a mi criterio, debería ser considerado. Intenté mostrar que desde el punto de vista del uso público de la razón, Kant piensa la legitimación por medio del concepto de Ilustración. Con esto, él reflexiona sobre la idea de la legitimación del poder y de la autoridad del Estado o de las instituciones religiosas fuera del Estado y de las instituciones religiosas, en una esfera pública de todos los posibles implicados ${ }^{21}$. Creo que Sieckmann debería tratar con más cuidado los papeles del Estado y del mercado en las decisiones jurídicas y políticas. En el ejemplo aludido sobre la prohibición de fumar en lugares públicos es necesario pensar en la acción y en los intereses de estas dos instituciones en el interior de la democracia ${ }^{22}$.

En segundo lugar, intenté analizar y contraponer las posiciones de Rousseau y Kant para sugerir cómo a partir de la confrontación de sus análisis del concepto de autonomía podríamos pensar en los conceptos de una autonomía pública y de una autonomía privada. En oposición a Habermas, considero que es posible ver estas dos vertientes en el interior de la filosofía práctica kantiana, en que tendríamos, por un lado, la deliberación pública y, por otro lado, la garantía de los

${ }^{21}$ Aquí sigo la tesis de Habermas en Transformaciones estructurales de la esfera pública en que el concepto de Öffentlichkeit gana terreno en la filosofía kantiana teniendo como contrapartida material la idea de una esfera pública de ciudadanos particulares que deliberan públicamente.

${ }^{22}$ Como el propio autor afirma, tendríamos la preocupación del Estado con la salud y la eficiencia de sus ciudadanos en contraposición a posiciones individuales que estarían vinculadas al puro placer de fumar. Sin embargo, son dos posiciones muy deferentes de poder. ¿Qué torna legítimo la posición de cada uno? Por otro lado, en el terreno de las hipótesis, habría también un gran interés de las industrias tabacaleras en esta discusión, habría lobby o propaganda. ¿Cómo pensar la legitimidad de una decisión teniendo en cuenta estas hipótesis? ¿Quién decidiría? 
Autonomía, legitimidad y legalidad: en torno de un antiguo conflicto...

derechos individuales, la idea de dignidad de la persona humana ${ }^{23}$. Esto no significa confundir el concepto de autonomía con el concepto de autonomía privada y, con esto, aproximar las posiciones de los dos filósofos a partir del presupuesto de los intereses privados del individuo. Por el contrario, se trata de pensar un doble valor al interior del concepto de autonomía que nos serviría como criterio para la legitimación del poder político: un elemento republicano y un elemento vinculado a las libertades civiles, o incluso, a los derechos humanos. La ventaja de esto es no necesitar presuponer la ficción liberal de un individuo que piensa y actúa de manera mecánica, es decir, de acuerdo solamente con sus propios intereses, sino por el contrario, presuponer un individuo que pueda cambiar sus posiciones en el interior del debate público. Por otro lado, impide también adoptar como única orientación una posición republicana más dura que no tendría en cuenta elementos de los derechos humanos.

Finalmente, me gustaría plantear una última consideración referida al debate contemporáneo sobre la cuestión de la legitimidad de la democracia. Creo que Sieckmann podría someter a prueba y aclarar su posición haciendo explícito con qué posiciones está debatiendo. Pretendí mostrar que su artículo se inicia con un presupuesto liberal duro que, al poco tiempo, va cediendo lugar a la idea de deliberación, ponderación de argumentos, intersubjetividad, etc. Intenté mostrar también como la mayoría de estos conceptos pueden tener su origen en el interior de la filosofía moderna, en especial en la de Kant y en la de Rousseau. Sin embargo, la filosofía contemporánea, incluso hasta los teóricos del derecho, están trabando un constante debate con estos autores ya clásicos y refinando sus posiciones por medio de esta discusión. ¿No sería tiempo entonces de que Sieckmann pensara en un camino semejante y, por lo tanto, estableciera una confrontación más clara con esta tradición?

${ }^{23}$ Sobre la crítica a Habermas acerca de no ver en el interior de la filosofía práctica kantiana el derecho y la ética como campos coexistentes, cf. Maus, I., Über Volkssouveränität: Elemente einer Demokratietheorie, Berlin, Suhrkamp, 2011. Acerca de la posición de Habermas, confrontar el capítulo tres de: Habermas, J. Faktizität und Geltung. Frankfurt-M., Suhrkamp, 1998. 
Maurício Keinert

\section{Bibliografía}

Bittner, R., "Máximas", Studia Kantiana, n. ㅇ 05, 2004.

Habermas, J. Faktizität und Geltung, Frankfurt, Suhrkamp, 1998.

Kant, I., Fundamentação da metafisica dos Costumes, San Pablo, Barcarolla, 2009.

Manin, B. "On Legitimacy and Political Deliberation". Political Theory. Vol. 15, $\mathrm{n}^{\circ} 3,1987$.

Maus, I., Über Volkssouveränität: Elemente einer Demokratietheorie, Berlin, Suhrkamp, 2011.

O’Neill, O. "Autonomy, Plurality and Public Reason", en Brender, N. y Krasnoff, L. (eds.), New Essays on the History of Autonomy, Cambridge, Cambridge University Press, 2004.

Rousseau, J.-J., Du Contrat Social, Paris, Pléiade, 1964, pág. 360. 